\title{
Multiphysics vibration FE model of piezoelectric macro fibre composite on carbon fibre composite structures
}

\author{
Yu Jia ${ }^{\mathrm{a}, *}$, Xueyong Wei ${ }^{\mathrm{b}}$, Liu Xu ${ }^{\mathrm{b}, \mathrm{a}}$, Congsi Wang ${ }^{\mathrm{c}}$, Peiyuan Lian ${ }^{\mathrm{c}}$, Song \\ $\mathrm{Xue}^{\mathrm{c}}$, Ahmed Al-Saadi ${ }^{\mathrm{a}}$, Yu Shi ${ }^{\mathrm{a}, *}$ \\ ${ }^{a}$ Department of Mechanical Engineering, University of Chester, Chester, CH2 4NU, UK \\ ${ }^{b}$ State Key Laboratory for Manufacturing Systems Engineering, Xi'an Jiaotong \\ University, 28 West Xianning Road, Xi'an 710049, China \\ ${ }^{c}$ Key Laboratory of Electronic Equipment Structure Design, Ministry of Education, \\ Xidian University, Xi'an 710071, China
}

\begin{abstract}
This paper presents a finite element (FE) model developed using commercial FE software COMSOL to simulate the multiphysical process of pieozoelectric vibration energy harvesting $(\mathrm{PVEH})$, involving the dynamic mechanical and electrical behaviours of piezoelectric macro fibre composite (MFC) on carbon fibre composite structures. The integration of MFC enables energy harvesting, sensing and actuation capabilities, with applications found in aerospace, automotive and renewable energy. There is an existing gap in the literature on modelling the dynamic response of PVEH in relation to real-world vibration data. Most simulations were either semi-analytical MATLAB models that are geometry unspecific, or basic FE simulations limited to sinusoidal analysis. However, the use of representative environment vibration data is crucial to predict practical behaviour for industrial development. Piezoelectric device physics involving solid mechanics and electrostatics were combined with electrical circuit defined in this FE model. The structure was dynamically excited by interpolated vibration data files, while orthotropic material properties for MFC and carbon fibre composite were individually defined for accuracy. The simulation results were validated by experiments with $<10 \%$ deviation, providing confidence for the proposed multiphysical FE model to design and optimise PVEH smart composite structures.
\end{abstract}

\footnotetext{
* Corresponding authors

Email addresses: yu.jia.gb@ieee.org (Yu Jia), y.shi@chester.ac.uk (Yu Shi)
} 
Keywords:

energy harvesting, MFC, CRFP, FE, multiphysics, COMSOL

\section{Introduction}

Composite materials, such as carbon fibre or glass fibre reinforced polymer, are increasingly desired in a number of applications such as aerospace, automotive, marine, infrastructural, renewable energy, biomedical and consumer products, due to their high ultimate strength, elastic moduli, high strength-to-weight-ratio and better failure reliability $[1,2,3]$. Furthermore, there are state-of-the-art development of multi-functional smart composite materials [4] that attempts to achieve the additional functionalities such as energy harvesting, structural health monitoring (SHM), condition sensing and wireless communication onto the otherwise purely mechanical material by integrating the multifunctional elements together.

In order to achieve this motivation, most researchers embedded SHM sensors within the composite materials [5] to acquire and process condition data of structures such as aircraft wings and wind turbine blades. This will help to detect early onset of potential failures, which will help to avoid catastrophic and costly consequences. However, either batteries or power cables are still required to power these sensors and electronic systems, which adds weight and regular maintenance or replacement costs [6]. On the other hand, the use of energy harvesting $(\mathrm{EH})$ technology $[6,7]$ has been developed as a potential self-powered sensing solution. Specifically, vibration energy harvesting (VEH) can be employed to convert ambient vibrational energy into useful electrical energy for the integrated sensors and electronics.

VEH technologies include a variety of transducers and scales, including macroscopic or mesoscale electromagnetic $[8,9,10,11]$ and piezoelectric $[12,13,14,15]$ generators to MEMS-scale electrostatic [16] and piezoelectric $[17,18,19]$ harvesters. Research efforts in the VEH field include attempts to increase the operational bandwidth $[20,21]$, operate at low frequency [22] or high temperature [23], improved electronic power conditioning efficiency $[24,25]$ or mechanical efficiency $[26,27]$. In relation to the integration with composite materials, piezoelectric films are particularly suitable due to the thin profile and manufacturing compatibility [28, 29]. A previous study [30] has shown experimental integration and characterisation of piezoelectric macro-fibre composite (MFC) transducers with CFRP targeted for aircrafts, especially in order to be compatible with the flexible design of aircraft wings. 
In order to achieve optimisation of design and system parameters for the integrated MFC on CFRP multi-functional composite structure, it is crucial to employ numerical and computational piezoelectric analysis. Most existing piezoelectric models are analytical or semi-analytical models based on beam theory $[31,32,13,33]$. While analytical models are useful to understand the underlying physics and to numerically simulate piezoelectric VEH systems in MATLAB, it is difficult to analysis designs other than the most basic topologies. Localised stress-strain distributions and the resultant charge generation was simplified and ignored in semi-analytical numerical models.

On the other hand, finite element (FE) models can help to analysis complex geometries when properly meshed. While existing FE models for piezoelectric VEH reported in the literature [27, 34] do exist, most of these FE models are either purely mechanical models or setup for simple sinusoidal simulations. Real world vibration is typically much more complicated than simple sine waves and can involve broadband noisy signals. The inclusion of piezoelectric transduction and electrical domain are also important, as both feedforward and feedback coupling effects with the mechanical domain need to be included in the analysis. Furthermore, when composite materials are involved, there is a further step in challenge to simulate anisotropic properties that can influence some of the vibrational modes. Therefore, a comprehensive vibration data driven piezoelectric VEH FE model, involving both mechanical and electrical domains, is needed to properly optimise and study designs across a wide range of topologies.

This paper proposes such a FE model for the first time, using COMSOL Multiphysics, to simulate the electrical and mechanical behaviour of an integrated MFC on CFRP composite structure. The multiphysical model combines solid mechanics, electrostatic and electrical circuit physic models within COMSOL, while employing eigenfrequency, frequency domain and time dependent model solvers. Response information such as mechanical stress, voltage output and power output across an impedance load can all be numerically predicted in both the time domain and frequency domain. Furthermore, interpolation of arbitrary wave input is used to feed representative environmental vibration data into the FE model. Measured vibration data from various sources, including aerospace, automotive, engine, bridge and rail applications, are used in this study. The proposed FE model can be used to simulate and undertake parametric studies for system optimisation of any piezoelectric VEH systems while using representative environment vibration data on top of basic sine wave excitations. 


\section{Analytical model}

The conventionally adopted dynamics model for base-pointed excited piezoelectric VEH involves a second order mass-spring damper system, which can be summarised by equation $1[32,35]$.

$$
\ddot{z}(t)+2 \zeta_{m} \omega_{0} \dot{z}(t)+\beta|\dot{z}| \dot{z}+\omega_{0}^{2} z(t)+\mu z^{3}(t)+\Theta v(t)=a(t)
$$

where, $z(t)$ is the displacement function, $\zeta_{m}$ is the mechanical viscous damping ratio, $\beta$ is the mechanical nonlinear damping ratio, $\omega_{0}$ is the natural frequency, $\mu$ is the mass normalised Duffing coefficient, $\Theta$ is the coupling coefficient from mechanical to electrical domain, $v(t)$ is the voltage generated from the piezoelectric transducer and $a(t)$ is the acceleration input driving the system. When the piezoelectric transducer is connected to an electrical circuit that draws electrical energy out of the device, equation 2 can be used to approximate the system behaviour.

$$
\ddot{z}(t)+2 \zeta_{m} \omega_{0} \dot{z}(t)+\beta|\dot{z}| \dot{z}+\omega_{0}^{2} z(t)+\mu z^{3}(t)+\frac{2 \zeta_{e} \omega_{0}^{3} Z_{i}}{E \sqrt{Z_{i}^{2}+Z_{\text {load }}^{2}}} z \dot{z}(t)=a(t)
$$

where, $\zeta_{e}$ is the electrical damping ratio, $Z_{i}$ is the internal impedance of the piezoelectric transducer typically dominated by its internal capacitance and $Z_{\text {load }}$ is the electrical load impedance.

Based on the linear piezoelectric theory [30], the mechanical stress and electric displacement field can be obtained by equations 3 and 4

$$
\begin{aligned}
& \sigma(t)=C \varepsilon(t)+e^{T} E \\
& D(t)=\epsilon_{P} E+e \varepsilon(t)
\end{aligned}
$$

where, $D$ is the electrical charge density displacement, $\epsilon_{P}$ is the dielectric permittivity of MFC, $E$ is the electric field, $e$ is the electromechanical coupling that can be expressed by the charge constant $d$ and composite stiffness $C(e=d \times C)$, and $\sigma$ and $\varepsilon$ represents the mechanical stress and strain respectively.

Depending on the poling direction and the direction of stress, charge constant of the piezoelectric material in different modes need to be used. For energy harvesting, it is typically $d_{31}$ mode where poling direction is along 
the thickness of the piezoelectric layer and stress is applied via tension and compression of the fibres during the bending mode. Charge constant $d$ is defined as the short circuit charge density per applied mechanical stress. Therefore, short circuit charge and current generated by the piezoelectric transducer can be summarised by equations 5 and 6 .

$$
Q_{s c}(t)=d_{31} \sigma_{a v} \int_{A} \mathrm{~d} A
$$

where, $Q_{s c}$ is the short circuit electrical charge, $\sigma_{a v}$ is the average stress experienced by the piezoelectric domain and $A$ is the active area of the piezoelectric domain.

$$
I_{s c}(t)=\omega(t) d_{31} \sigma_{a v} A
$$

where, $I_{s c}$ is the short circuit current and $\omega(t)$ is the frequency at which the piezoelectric transducer is being excited at. With a given electrical impedance load $Z_{\text {load }}$ to extract electrical energy, the generated current through the load $I_{\text {load }}$ can be represented by equation 7 and the electrical power $P(t)$ can be calculated by equation 8 .

$$
\begin{gathered}
I_{\text {load }}(t)=\frac{I_{s c}^{2}(t) Z_{i}}{\sqrt{Z_{i}^{2}+Z_{\text {load }}^{2}}} \\
P(t)=I_{\text {load }}^{2}(t) Z_{\text {load }}=\frac{I_{\text {sc }}^{2}(t) Z_{\text {load }} Z_{i}^{2}}{Z_{i}^{2}+Z_{\text {load }}^{2}}
\end{gathered}
$$

\section{Finite element model}

\subsection{Model}

The design studied here involves a piezoelectric macro-fibre composite (MFC) energy harvesting transducer (M8525-P2 from Smart Material) integrated on a carbon fibre composite beam through co-curing. The co-curing process was developed in previous work $[30,36]$. The specific example device investigated here was manufactured from 8 plies of epoxy resin infused carbon fibre prepreg $(0.23 \mathrm{~mm})$. The carbon fibre reinforced polymer (CFRP) beam was $60 \mathrm{~mm}$ in width and $200 \mathrm{~mm}$ in length. The MFC transducer was placed close to the clamped end of the CFRP beam in order to harness the most active stress region. Figure 1 summarises the employed geometry and figure 2 summarises the finite element (FE) model. 


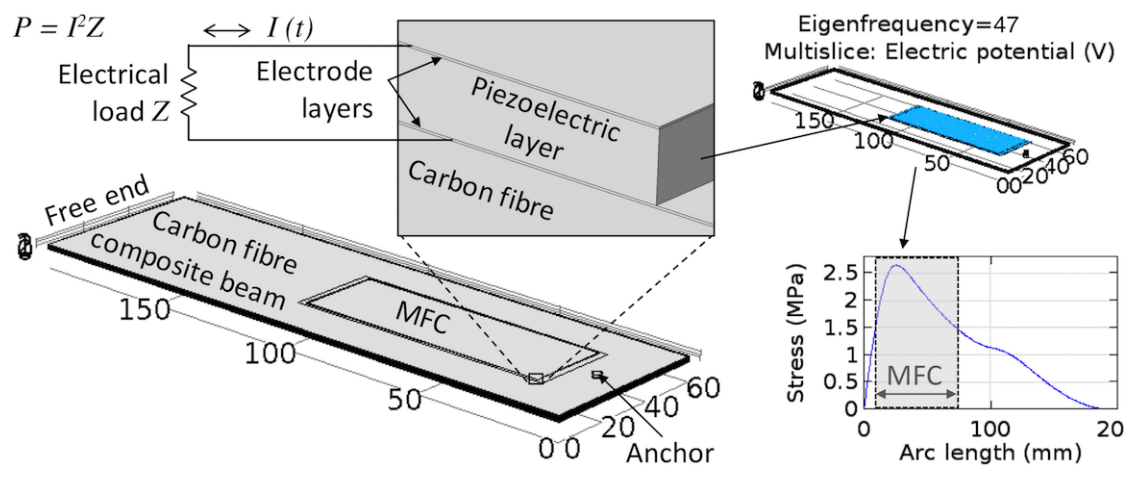

Figure 1: Schematic of FE model used to simulate the MFC transducer on carbon fibre composite beam structure. Electrical power output $P$ across an electrical load $Z$ is calculated from the current $I$ generated from the MFC as the beam is mechanically strained.

Within COMSOL Multiphysics, Piezoelectric device multiphysics was employed, which involved a combination of Solid Mechanics and Elestrostatics physics in order to model both the mechanical and piezoelectric behaviour. Electrode layers sandwiched the MFC generator and Electrical Circuit physics was used to model a resistance load across the MFC terminals.

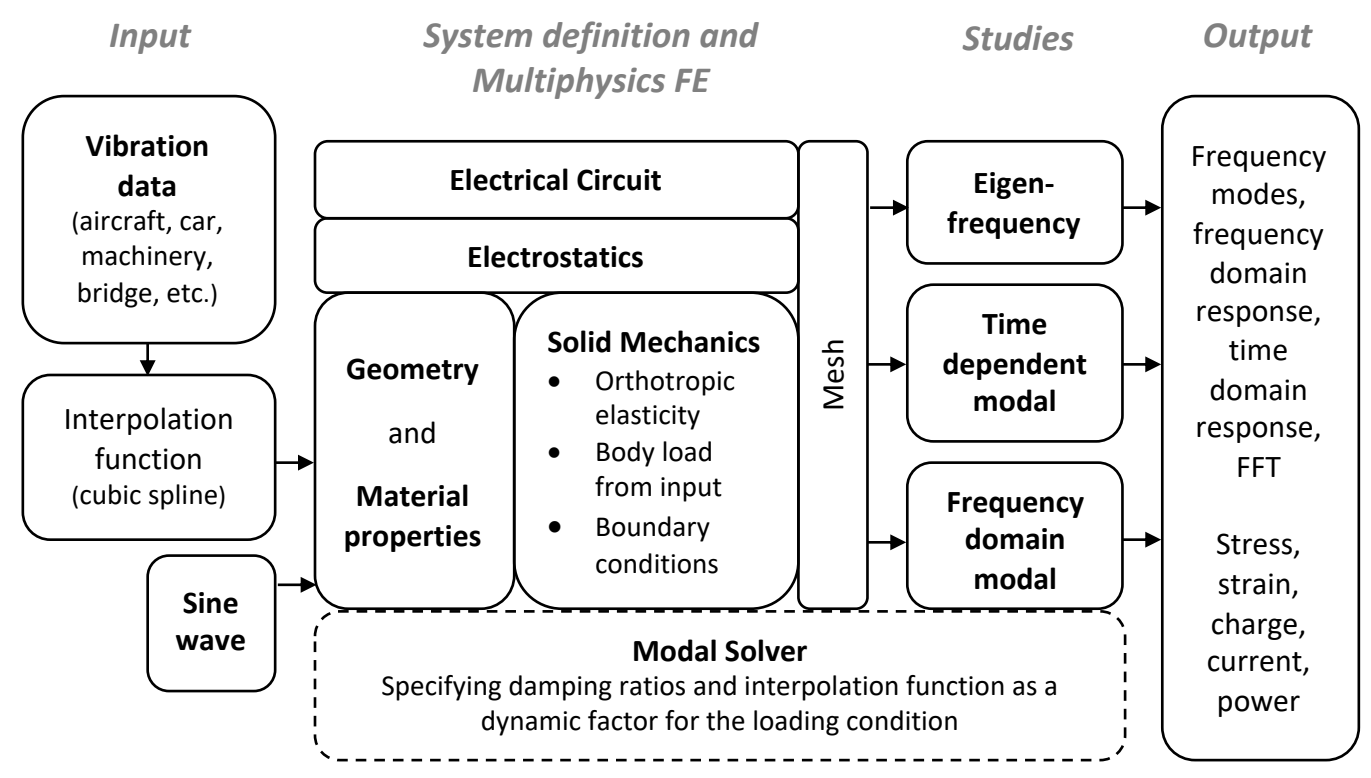

Figure 2: Summary of the multiphysics FE model setup in COMSOL to simulate the mechanical and electrical responses of the piezoelectric MFC on carbon fibre composite beam when subjected to vibrational data input. 
In terms of FE studies, Eigenfrequency, Time dependent modal and Frequency domain modal were employed. This helps to solve the resonant frequency values and mode shapes, time domain and FFT responses, and frequency domain responses, respectively. Both sinusoidal input and vibration data were used as the dynamic loading on the device. Samples of representative environment vibration data from various applications (including aerospace, automotive, engine, bridge and rail) were imported into COMSOL using interpolation function.

The time domain data was interpolated using piecewise cubic spline function in order to resolve the localised time domain features. However, it should be noted that vibration features will always lose some information when undergoing any step of mathematical manipulation and/or transformation. The interpolation functions can only approximate the original vibration signal. Furthermore, the resolution of the vibration signal is also limited by the sampling rate and applied filters when the original data was logged.

The vibration data interpolation function is then used to act as the dynamic loading factor within the Modal Solver in order to vary the acceleration body load $a(t)$ applied on the device. Damping ratios for both the mechanical and electrical domains can be applied within the Solid Mechanics definitions and the Modal Solver. Fitted quality factor $Q$ values from experimental data was used to determine the damping ratio in the FE model.

The FE model can output any information solved by the 3 employed physics, giving rise to an array of mechanical and electrical responses. This included displacement, stress, strain and energy information, as well as electrical charge, voltage and power generated across the impedance load. Eigenfrequency study revealed the various resonant frequency and mode shape information, but its amplitude response is arbitrary. While the two modal studies provided absolute amplitude information in either the time domain and frequency domain. Therefore, the modal studies can advise on the average power achievable from the device for various vibration input traces.

Figure 3 provides and example of eigenfrequency mechanical stress and electrical voltage responses for the first bending resonant mode at $47.2 \mathrm{~Hz}$. The line graphs show that the high stress region of the CFRP beam is harvested by the MFC. The generated voltage is a result of dynamic stress rather than static loading. For instance, if a beam is statically stressed, voltage output would return to zero after the initial step response. Henceforth, the study of time domain response is crucial to determine the average response rather than simply the instantaneous peak values. 

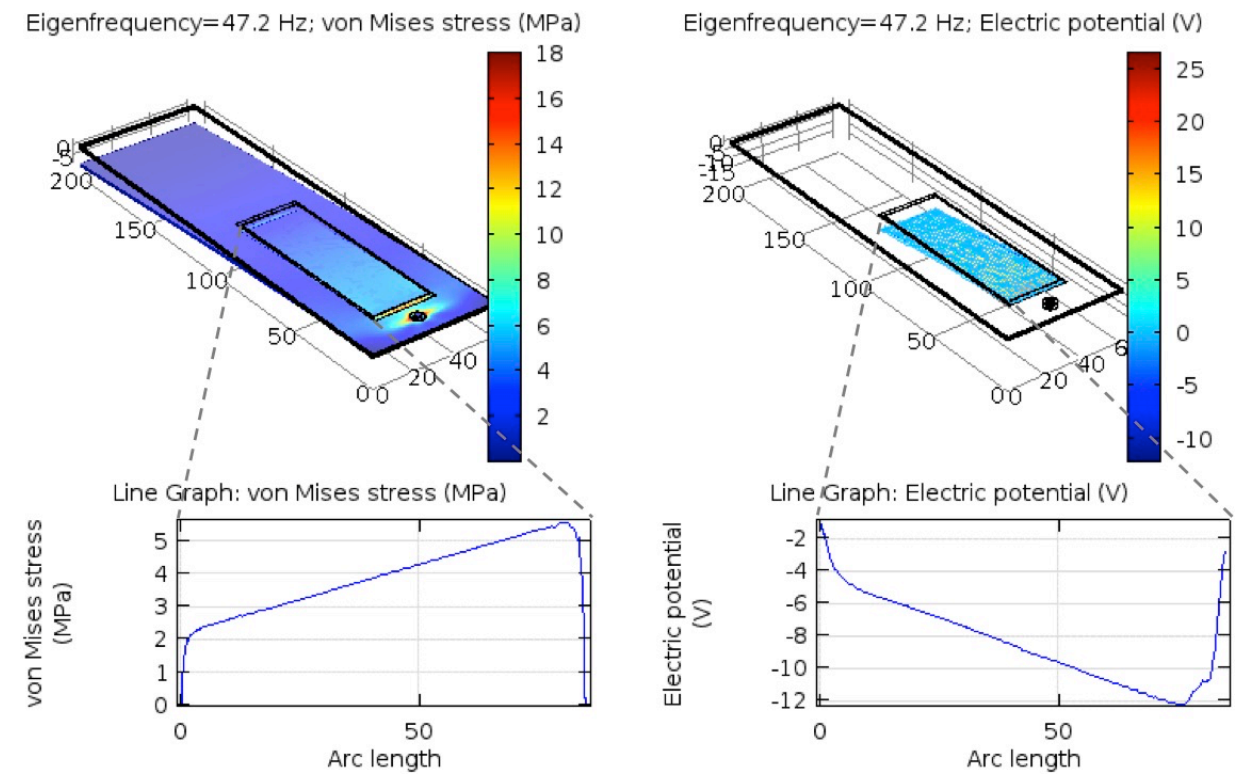

Figure 3: The FE simulated mechanical and electrical responses of the MFC element when the beam is driven at the first resonant mode.

\subsection{Material properties and orthotropic elasticity}

Unlike the isotropic nature of metallic and amorphous materials, composite materials are orthotropic. Therefore, it is important to fully define the 3-dimensional linear elastic properties for the CFRP and MFC. Table 1 summarises the key material property definitions for the employed RC200T carbon fibre, while table 2 summarises the key material property definitions for the MFC 8525-P2 transducer.

Table 1: Carbon fibre composite (RC200T) orthotropic elasticity definitions.

\begin{tabular}{c|rrr}
\hline \multirow{2}{*}{ Young's modulus (GPa) } & $E_{x}$ & $E_{y}$ & $E_{z}$ \\
& 59.45 & 60.3 & 3.9 \\
\hline \multirow{2}{*}{ Shear modulus (GPa) } & $G_{x y}$ & $G_{y z}$ & $G_{x z}$ \\
& 62.9 & 1.5 & 62.35 \\
\hline \multirow{2}{*}{ Poisson's ratio (1) } & $\nu_{x y}$ & $\nu_{y z}$ & $\nu_{x z}$ \\
& 0.3 & 0.4 & 0.3 \\
\hline \hline Density $\rho\left(\mathrm{kg} / \mathrm{m}^{3}\right)$ & \multicolumn{3}{|c}{1,800} \\
\hline
\end{tabular}

The elastic properties determine the mechanical strength and behaviour, while the piezoelectric and dielectric properties determine the mechanical- 
Table 2: Macro fibre composite properties [30, 37]. MFC 8525-P2 ( $d_{31}$ mode) was employed as the transducer for piezoelectric vibration energy harvesting.

\begin{tabular}{l|r}
\hline \multicolumn{2}{c}{ Mechanical properties } \\
\hline Young's modulus $E_{1}$ in rod direction $(\mathrm{GPa})$ & 30.34 \\
Young's modulus $E_{1}$ in electrode direction $(\mathrm{GPa})$ & 15.86 \\
Shear modulus $G_{12}(\mathrm{GPa})$ & 5.52 \\
Poisson's ratio $\nu_{12}(1)$ & 0.31 \\
Poisson's ratio $\nu_{21}(1)$ & 0.16 \\
Density $\left(\mathrm{kg} / \mathrm{m}^{3}\right)$ & 5,400 \\
\hline \multicolumn{2}{c}{ Piezoelectric and dielectric properties } \\
\hline Charge constant $d_{31}(\mathrm{pC} / \mathrm{N})$ & -170 \\
Charge constant $d_{33}(\mathrm{PC} / \mathrm{N})$ & 400 \\
Capacitance per unit area $\left(\mathrm{nF} / \mathrm{cm}^{2}\right)$ & 7.8 \\
Dielectric permittivity $\epsilon_{p}(\mathrm{nF} / \mathrm{m})$ & 0.15 \\
Relative permittivity $\epsilon_{r}(1)$ & 1,695 \\
\hline
\end{tabular}

to-electrical conversion. Typically, for piezoelectric transducers, power correlates directly with the piezoelectric charge constant $d$ (short circuit charge density per applied mechanical stress) and inversely correlated with relative permittivity or dielectric constant $\epsilon_{r}$. MFC is made from lead zirconate titanate (PZT) fibres, so its piezoelectric and dielectric properties are typical of bulk PZT, despite its high flexibility.

The Eigenfrequency study was used to reveal up to the first 25 resonant modes, ranging from $47.2 \mathrm{~Hz}$ (1st bending mode) to $8325.9 \mathrm{~Hz}$ (14th torsional mode). Within this range, there were 9 bending modes, 2 lateral modes and 14 torsional modes. Figure 4 illustrates the first few resonant mode shapes with stress contour. Typically, only the first bending mode is ideally suited for vibration energy harvesting, as all the higher modes would result in partial charge cancellation from opposing stress regions across the single MFC piezoelectric transduction element.

While excited at the vicinity of the first bending mode, resonant amplification and accumulation of vibrational energy can be achieved. However, even at higher frequency excitations as well as shocks across the wider spectrum, non-resonant capture of vibrational energy, albeit at significantly reduced levels, can still be achieved. Real world vibration are typically noisy, broadband and contain shock impulses, which will result in step response oscillatory decay at the first resonant frequency value and mode shape. 
Eigenfrequency $=47.2 \mathrm{~Hz}$, stress $(\mathrm{MPa})$

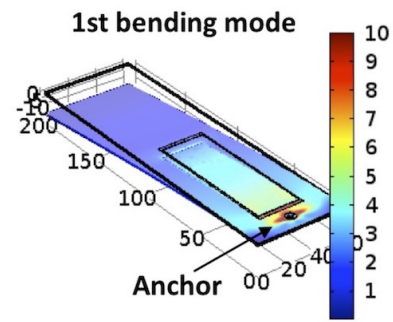

Eigenfrequency $=362 \mathrm{~Hz}$, stress $(\mathrm{MPa})$

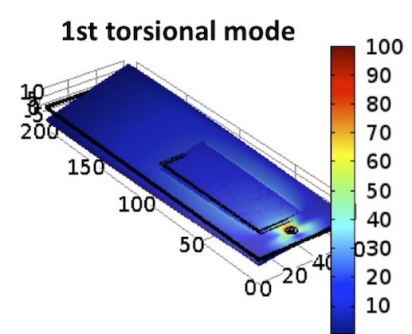

Eigenfrequency $=275 \mathrm{~Hz}$, stress $(\mathrm{MPa})$

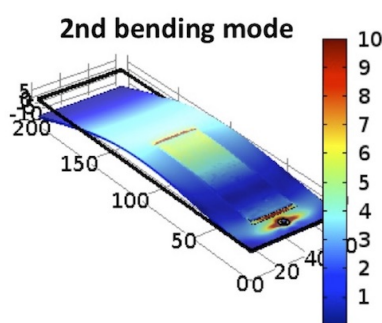

Eigenfrequency $=1095 \mathrm{~Hz}$, stress $(\mathrm{MPa})$

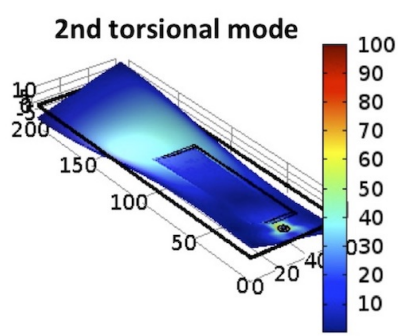

Eigenfrequency $=772 \mathrm{~Hz}$, stress $(\mathrm{MPa})$

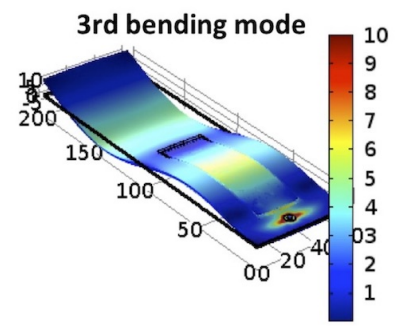

Eigenfrequency $=147 \mathrm{~Hz}$, stress $(\mathrm{MPa})$

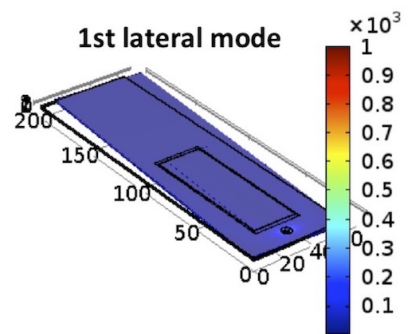

Figure 4: The first few mode shapes for bending, torsional and lateral resonant modes simulated using orthotropic elasticity.

Table 3: Comparison of resonant frequencies for various modes between experimental measurement, FE simulation using isotropic modulus of elasticity (single $E, \nu$ and $G$ ) and orthotropic elasticity for composite material (3D compliance matrix for $E, \nu$ and $G)$. Experimental prototype deviated away from the target design parameters due to manufacturing tolerance. Analytical calculation for the first bending mode using EulerBernoulli equation revealed $46.5 \mathrm{~Hz}$ and using Timoskenko equation revealed $47.2 \mathrm{~Hz}$. Therefore, FE model with orthotropic property definition is more accurate.

\begin{tabular}{c|r|rr|rr}
\hline \multirow{2}{*}{ Mode } & \multicolumn{2}{|c|}{ Measured } & \multicolumn{2}{|c|}{ FE isotropic } & \multicolumn{2}{c}{ FE orthotropic } \\
& freq. (Hz) & freq. (Hz) & error (\%) & freq. (Hz) & error (\%) \\
\hline 1st bending & 46.0 & 46.0 & 0.0 & 47.2 & +2.6 \\
2nd bending & 272 & 271 & -0.4 & 275 & +1.1 \\
3rd bending & 764 & 762 & -0.3 & 772 & +1.0 \\
4th bending & 1,489 & 1,488 & -0.1 & 1,499 & +0.7 \\
5th bending & 2,290 & 2,265 & -1.1 & 2,380 & +3.9 \\
\hline 1st torsional & 358 & 270 & -24.6 & 362 & +1.1 \\
2nd torsional & 1,047 & 780 & -25.5 & 1,095 & +4.6 \\
3rd torsional & 1,897 & 1,387 & -26.9 & 1,935 & +2.0 \\
4th torsional & 2,790 & 2,033 & -27.1 & 2,843 & +1.9 \\
\hline 1st lateral & 145 & 132 & -9.0 & 147 & +1.4 \\
\hline
\end{tabular}


Table 3 summarises the comparison of experimentally measured frequency values and FE simulated results. Hypothetical isotropic values are assumed for comparison purposes. This table illustrates the importance of orthotropic elasticity definitions within the composite material in order to achieve good accuracy (deviation $<5 \%$ ). On the other hand, a basic isotropic model can only be used for single direction bending modes and significantly deviates for the torsional and lateral modes. Therefore, while a basic isotropic model might have been sufficient for sinusoidally excited scenarios near the first bending mode, any response from broadband vibrational signals must observe orthotropic elasticity definitions in order to accurately simulate higher frequency and non-resonant responses across the wider spectrum.

Furthermore, isotropic results on first instance seem to match up with measured results for the bending modes. However, this is because the FE accuracy with isotropic material property definitions happens to deviate in the same direction as the manufacturing tolerance of the practical device used in the experimental validation here. In comparison with analytical calculations evidenced in the results section, it can seen that the FE model using the full orthotropic material properties are more accurate. For the first bending mode, analytically calculated resonant frequency using the basic Euler-Bernoulli equation revealed $46.5 \mathrm{~Hz}$ and using the more comprehensive Timoskenko equation revealed $47.2 \mathrm{~Hz}$.

\subsection{Vibration data analysis}

Figure 5 illustrates an example of the FE simulation, with $2 \mathrm{kHz}$ broadband aerospace vibration data used as the excitation input, and showing mechanical strain, voltage and power output in time domain. FFT of time domain power output is also shown, where the response peaks accumulate towards the 1st bending mode. The vibration input was based on typical

aircraft vibration while in-flight [38], where the average amplitude was $14 \mathrm{~g}$ and the frequency response closely follows a band-limited $2 \mathrm{kHz}$ white noise. It can be seen that from the shock based dynamic mechanical response, a relatively higher frequency electrical response is generated from the small wave dynamic oscillations sitting on top of the strain response.

A series of various vibration data was also used, measured directly from representative environments using Gulf Coast Data Concepts accelerometer data loggers. This includes vibration of CGJ aircraft chassis in figure 6, BMW 525d engine beneath the bonnet in figure 7, 1,560 RPM compressor engine in figure 8, a cross girder from the Forth Road suspension bridge in 
Input
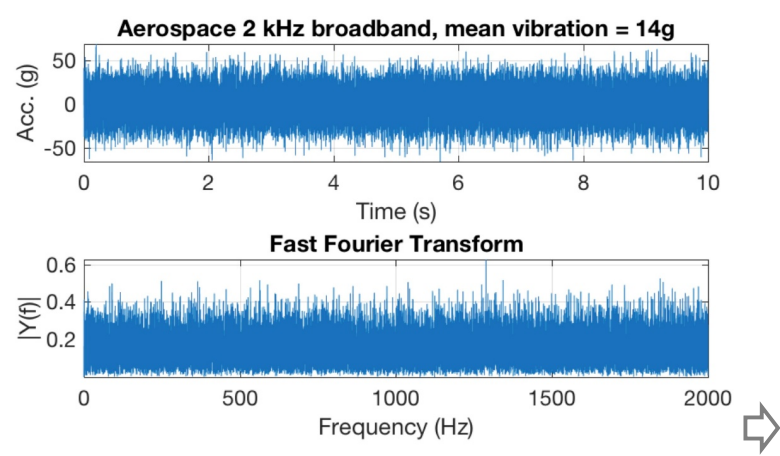

Spectrogram with Hamming window size $=\mathbf{5 1 2}$

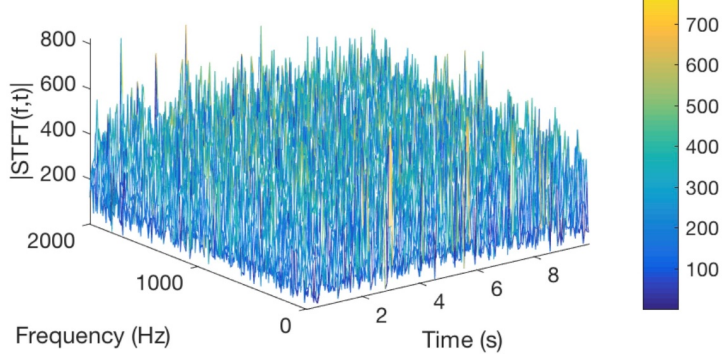

Output
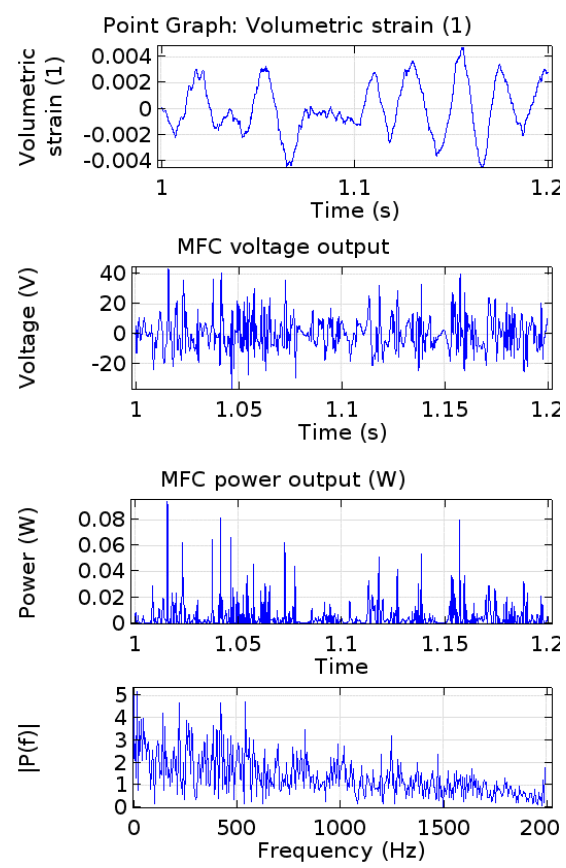

Figure 5: An example of using representative aerospace in-flight vibration data (broadband $2 \mathrm{kHz}$ ) as dynamic loading in the FE model. Mechanical and electrical outputs from the piezoelectric device is computed, including stress, strain, voltage and power across a load resistance of $20 \mathrm{k} \Omega$.

figure 9 , section of a rail track above vibration absorbers in figure 10 and inside the cabin of a rail carriage in figure 11.

Time domain data traces of these application based environmental vibration data were processed and analysed using FFT and STFT in MATLAB. For all these vibration traces, none of them are optimally suited for the 47.2 $\mathrm{Hz}$ first resonant mode of the device embodiment investigated here. So the harvester will primarily operate in non-resonant mode and experience shockinduced step responses. Selected traces were in turn imported into COMSOL using the established interpolation function and used for the Time Dependent Modal study. FFT was carried out on the time domain response from the FE simulation in order to assess the frequency characteristics. 

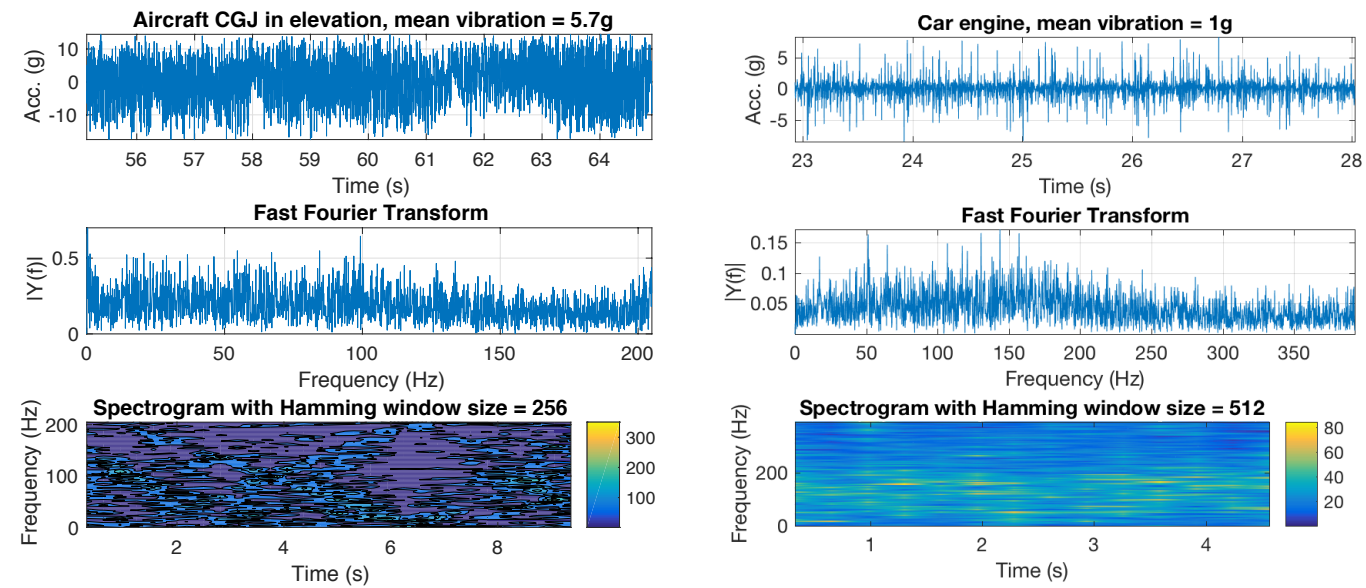

Figure 6: CGJ aircraft vibration data used as excitation for the FE model.

Figure 7: BMW 525d car engine vibration data used as excitation for the FE model.
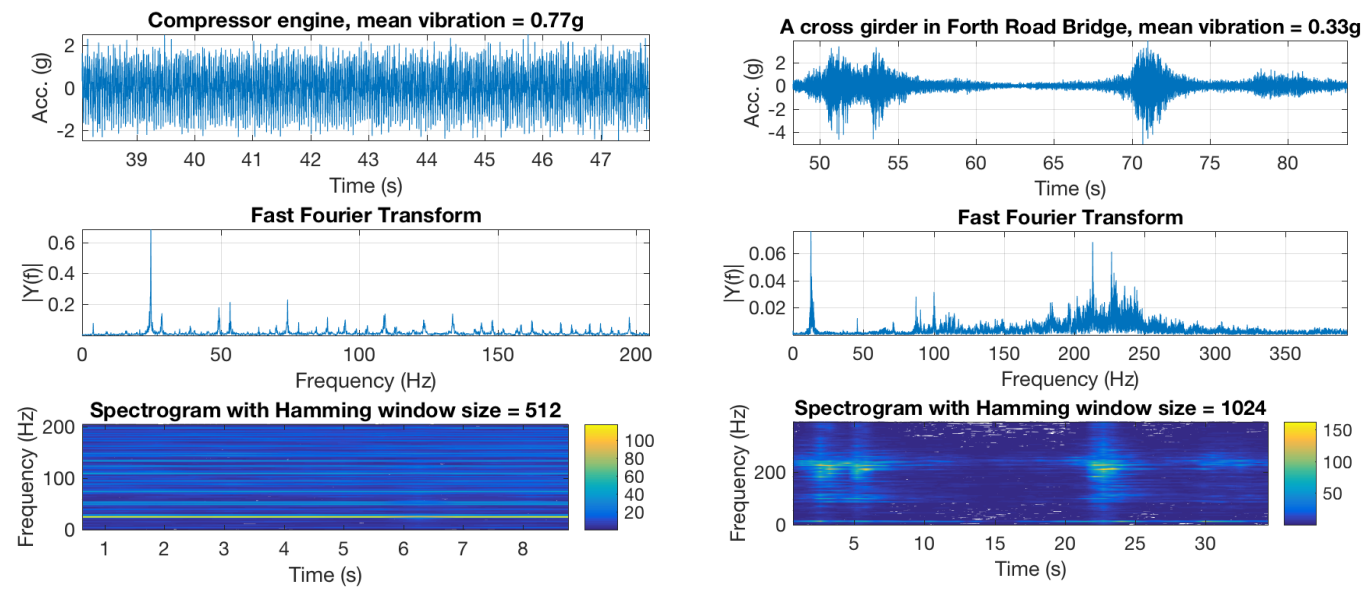

Figure 8: Compressor engine vibration data used as excitation for the FE model.

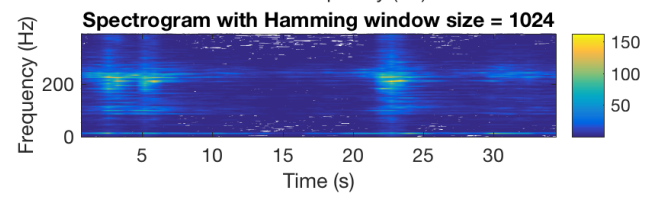

Figure 9: Suspension bridge vibration data used as excitation for the FE model.

\subsection{Experimental validation}

MFC-on-CFRP prototype device was manufactured and experimentally characterised for model validation. The full integration process $[30,36]$ and experimental characterisation [36] was reported previously. Figure 12 presents a photograph of the device mounted on an electrodynamic shaker. Thin electrical wires were attached using conductive silver adhesive epoxy. The electrical leads are connected to an impedance box and measured on a digital oscilloscope. Accelerometer was attached at the base point in order to 

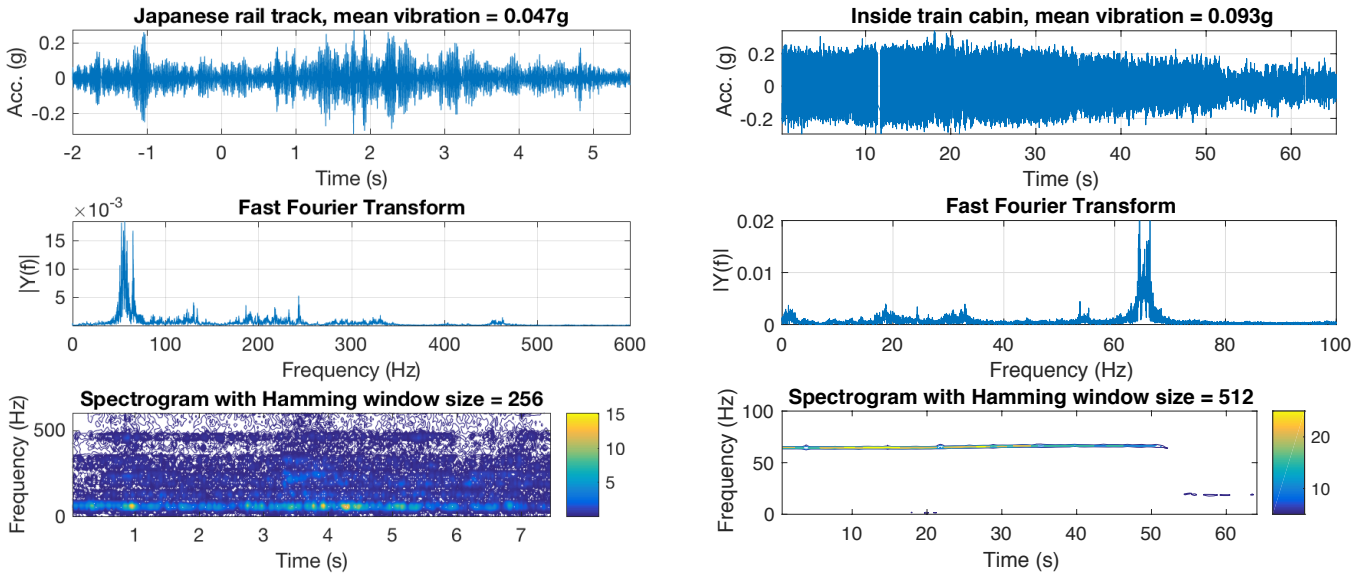

Figure 10: Rail track vibration data used as excitation for the FE model.

Figure 11: Train cabin interior vibration data used as excitation for the FE model.

control the acceleration amplitude and frequency of the base point excitation fed into the harvester device.

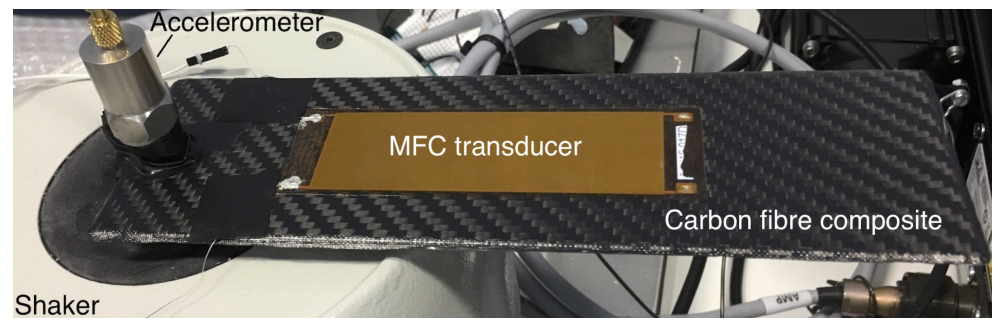

Figure 12: Integrated MFC-on-CFRP prototype used to validate the FE model. The full experimental characterisation was previously studied [36].

A summary of the experimental setup used for measurement and characterisation is presented in figure 13. Both sine wave excitation and vibration data traces are used to drive the electrodynamic shaker. Agilent Waveform Editor was used to communicate vibration data to an Agilent function/arbitrary wave generator, which in turn programmed the vibration from the BKSV LDS electrodynamic shaker. 


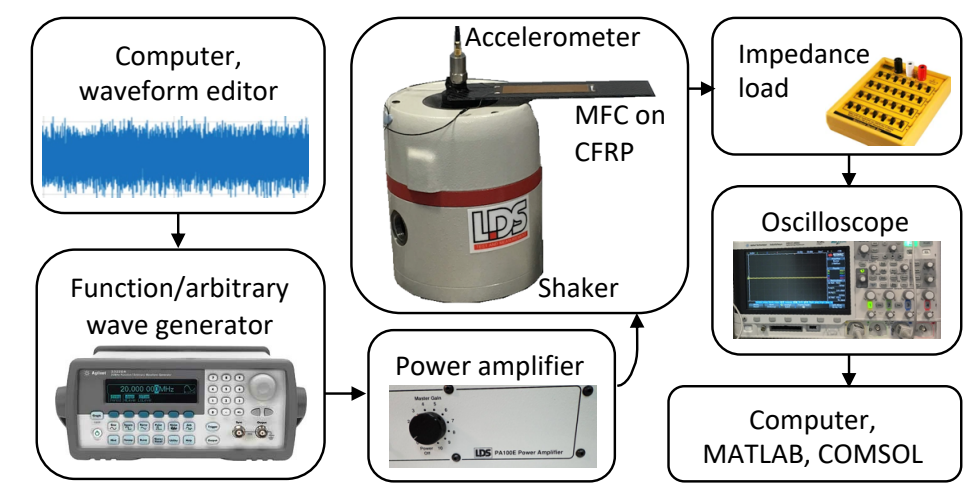

Figure 13: Experimental setup used to test the integrated MFC-on-CFRP prototype.

\section{Results and discussion}

\subsection{Sinusoidal response}

At first instance, sine wave excited resonant response was characterised. Figure 14 compares the experimentally measured power output in the frequency domain for the manufactured prototype and the simulated result. The experimentally measured quality factor was used to fit the damping ratio for the FE model in order to achieve alignment. Power $P(t)$ values are calculated from RMS voltage $V(t)$ generated across a load resistance $R$, using $P(t)=V^{2}(t) / R$.

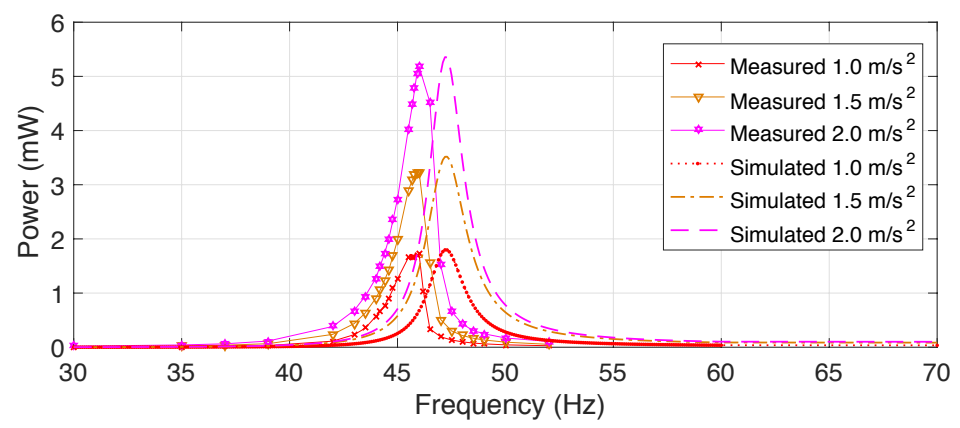

Figure 14: Comparison of experimentally measured and simulated frequency domain response for 1st bending resonant mode. Resonant frequency deviation was $2.6 \%$.

Table 4 compares the first resonant mode frequency computed by experimental analytical and FE simulation. While the classic method of analytical eigenfrequency calculation is based on the Euler-Bernoulli beam theory, 
such an approach is primarily suitable for isotropic materials only. On the other hand, when dealing with orthotropic composite beams, Timoshenko beam theory needs to be applied [39]. The Timoshenko frequency equation for composite beams used here was derived and algebraically expanded by Banerjee (2001) [39].

Table 4: First eigenfrequency (first bending mode) comparison of the experimental measurement, analytical calculations (classic beam theory and Timoskenko beam thoery) and FE simulation using orthotropic material property definitions.

\begin{tabular}{ll|r}
\hline Method & Frequency $(\mathrm{Hz})$ \\
\hline Experimental measurement & 46.0 \\
Analytical: & Euler-Bernoulli & 46.5 \\
Analytical: & Timoskenko & 47.2 \\
Numerical: & FE simulation & 47.2 \\
\hline
\end{tabular}

The measured first bending mode's resonant frequency when loaded with a matched impedance $(20 \mathrm{k} \Omega$ ) was at $46.0 \mathrm{~Hz}$, while that for the FE simulation was $47.2 \mathrm{~Hz}$ with a small deviation of $+2.6 \%$. The deviation was most likely due to fabrication tolerance, tolerance associated with various material properties and experimental measurement uncertainty. Furthermore, based on the frequency domain characteristics shown in figure 14 for the experimentally measured results, there seems to be a degree of nonlinear asymmetry for the resonant peaks. This behaviour suggests the presence of Duffing type nonlinearity [40] represented by a cubic spring term. More specifically, the bending of the resonant peak towards the higher frequency signifies spring hardening nonlinearity. This nonlinearity is significant even for small amplitude response, which further suggests that the source of this cubic nonlinearity is inherent in nature. The topology of the device is that of a basic cantilever, which implies geometric nonlinearity should be minimal. However, as the MFC and carbon fibre plies were co-cured, the differential thermal expansion coefficient can potentially result in non-trivial residual stress in the final beam. Therefore, this residual thermal stress could be a major contributing cause to this weakly nonlinear behaviour.

Table 5 compares the average resonant power output between experimentally measured result and simulated result when subjected to sinusoidal excitation at varying acceleration levels. This is for resonant power output for the 1st bending mode. Due to the slightly different resonant frequency value for the experimental prototype and $\mathrm{FE}$ value, excitation frequencies in 
experiment and in simulation were $46.0 \mathrm{~Hz}$ and $47.2 \mathrm{~Hz}$ respectively. Simulation deviated from experimental measurement by $<10 \%$.

Table 5: Comparison of power output between experimental measurement (or fitted values for large acceleration $>3 \mathrm{~g}$ ) and FE simulation for sinusoidal excitations.

\begin{tabular}{cc|r|r|r}
\hline $\begin{array}{c}\text { Vibration } \\
\text { input }\end{array}$ & $\begin{array}{c}\text { Acc. } \\
(\mathrm{g})\end{array}$ & $\begin{array}{r}\text { Experimental } \\
\text { mean power }(\mathrm{mW})\end{array}$ & $\begin{array}{r}\text { FE simulated } \\
\text { mean power }(\mathrm{mW})\end{array}$ & $\begin{array}{r}\text { Deviation } \\
(\%)\end{array}$ \\
\hline Sine, 1st & 0.10 & 1.72 & 1.80 & +4.7 \\
bending & 0.15 & 3.24 & 3.52 & +8.6 \\
mode & 0.20 & 5.18 & 5.36 & +3.5 \\
\hline
\end{tabular}

\subsection{Vibration data response}

In addition to sinusoidal excited piezoelectric FE simulation that has previously been reported in the literature [34], this paper uniquely studies FE simulation incorporating representative environment vibration data for the various applications. Results were attained from both FE simulation and experimental validation. Waveform editor on a computer and arbitrary waveform generator were used to experimentally programme the electrodynamic shaker using the vibration data files. Frequency and acceleration information from the vibration traces were equalised (or scaled) using accelerometers. In FE simulation, interpolation functions were used to import the vibration data files (either csv or txt) and dynamically load the model.

Table 6 summarises the experimentally measured and FE simulated average power output for the MFC-on-CFRP prototype device. The electrodynamic shaker is limited in maximum shuttle travel and therefore the attainable acceleration amplitude. For vibration traces greater than $3 \mathrm{~g}$ of mean acceleration, experimental data points collected for lower scaled acceleration levels were used to construct the trend line and predict the power output from the fitted graph.

In both cases, average power output was taken across a load resistance of $20 \mathrm{k} \Omega$. This is the matched impedance load for the first resonant response, but might not necessarily be the optimal resistance for the varying vibration inputs across the frequency spectrum. Though, for consistency in order to enable comparison, this load resistance was fixed. For realistic applications, a dynamic control system needs to be employed to enable maximum power point tracking, which is available with commercial power conditioning elec- 
Table 6: Comparison of power output between experimental measurement (or fitted values for large acceleration $>3 \mathrm{~g}$ ) and FE simulation for various vibration data input. Deviation is within $\pm 10 \%$ except for outlier cases with power values $<0.1 \mathrm{~mW}$ where background noise is relatively more significant.

\begin{tabular}{cc|r|r|r}
\hline $\begin{array}{c}\text { Vibration } \\
\text { input }\end{array}$ & $\begin{array}{c}\text { Acc. } \\
(\mathrm{g})\end{array}$ & $\begin{array}{r}\text { Experimental } \\
\text { mean power }(\mathrm{mW})\end{array}$ & $\begin{array}{r}\text { FE simulated } \\
\text { mean power }(\mathrm{mW})\end{array}$ & $\begin{array}{r}\text { Deviation } \\
(\%)\end{array}$ \\
\hline Aerospace & 1.6 & 1.05 & 0.96 & -8.6 \\
in-flight & 14 & 7.8 & 8.09 & +3.7 \\
\hline Aerospace & 1.6 & 0.97 & 0.90 & -7.2 \\
in elevation & 5.7 & 2.0 & 1.82 & -9.0 \\
\hline Road car & 0.25 & 0.37 & 0.35 & -5.4 \\
\hline Compressor & 1.0 & 0.077 & 0.06 & -22.1 \\
\hline Bridge & 0.32 & 0.17 & 0.18 & +5.9 \\
\hline Trail track & 0.047 & 0.012 & 0.018 & +50.0 \\
\hline Train cabin & 0.093 & 0.045 & 0.062 & +37.8 \\
\hline
\end{tabular}

tronic chips such as LTC3588 that is popularly used for vibration energy harvesting $[11,41]$.

Responses are typically off-resonance, non-resonant and shock-induced step responses. It can be noted that the FE simulated results deviated from the experimental measurement by less than $\pm 10 \%$ for all scenarios where the average power output is $>0.1 \mathrm{~mW}$. However, for the 3 outlier cases (compressor, train track and train cabin) where the power values are $<0.1 \mathrm{~mW}$, deviation was more significant. This could be because for these small power output cases, background noise, tolerance and measurement uncertainties have more substantial influences. Therefore, reliability of model prediction at low power levels drops due to the background noise. This suggests that when system parameters are optimally tuned to enable resonant-amplified power generation, the results from FE simulation and the experimental counterparts should converge.

\section{Conclusion}

This paper uniquely presents a dynamic multiphysics finite element model that uses representative environment vibration data to dynamically load a piezoelectric MFC on carbon fibre composite beam. The resultant mechanical response from the beam and the electrical output from the piezoelectric transducer were simulated. The FE model was experimentally validated for 
both sinusoidal excitations and vibration data files for a variety of applications including aerospace, automotive, engine, bridge and rail. The FE model deviated from the experimental measurement by $<10 \%$ in terms of resonant frequencies and average power output. Therefore, the proposed multiphysical FE model can enable parametric study for any topological designs and vibration spectra, in order to employ computational analysis for system parameter optimisation during the design stage of vibration powered smart composite strcutures.

\section{Acknowledgements}

This research was supported by Innovate UK (Project Reference 104030). Original vibration data collection and measurement was undertaken here.

\section{References}

[1] Y. Shi, T. Swait, C. Soutis, Modelling damage evolution in composite laminates subjected to low velocity impact, Composite Structures 94 (9) (2012) 2902-2913.

[2] Q. Liu, X. Xu, J. Ma, J. Wang, Y. Shi, D. Hui, Lateral crushing and bending responses of cfrp square tube filled with aluminum honeycomb, Composites Part B: Engineering 118 (2017) 104-115.

[3] R. M. Jones, Mechanics Of Composite Materials, Taylor \& Francis, New York, NY, 1999.

[4] R. F. Gibson, A review of recent research on mechanics of multifunctional composite materials and structures, Composite Structures 92 (12) (2010) 2793-2810.

[5] K. J. Narayana, R. Gupta Burela, A review of recent research on multifunctional composite materials and structures with their applications, Materials Today: Proceedings 5 (2, Part 1) (2018) 5580-5590.

[6] S. Priya, D. Inman, Energy Harvsting Technologies, Springer US, New York, 2009.

[7] S. Anton, H. Sodano, A review of power harvesting using piezoelectric materials (2003-2006), Smart Mater. Struct. 16 (3) (2007) R1-R21. 
[8] S. P. Beeby, R. N. Torah, M. J. Tudor, P. Glynne-Jones, T. O’Donnell, C. R. Saha, S. Roy, A micro electromagnetic generator for vibration energy harvesting, J. Micromech. Microeng 17 (7) (2007) 1257.

[9] Y. Jia, J. Yan, K. Soga, A. Seshia, A parametrically excited vibration energy harvester, J. Intel. Mat. Syst. Str. DOI: 10.1177/1045389X13491637.

[10] Z. Li, L. Zuo, G. Luhrs, L. Lin, Y. x. Qin, Electromagnetic energy-harvesting shock absorbers: Design, modeling, and road tests, IEEE Transactions on Vehicular Technology 62 (3) (2013) 1065-1074. doi:10.1109/TVT.2012.2229308.

[11] Y. Jia, J. Yan, S. Du, T. Feng, P. Fidler, C. Middleton, K. Soga, A. A. Seshia, Real world assessment of an auto-parametric electromagnetic vibration energy harvester, Journal of Intelligent Material Systems and Structures 29 (7) (2017) 1481-1499.

[12] S. Roundy, P. Wright, A piezoelectric vibration based generator for wireless electronics, Smart Mater. Struct. 13 (5) (2004) 1131-1142.

[13] A. Erturk, D. Inman, An experimentally validated bimorph cantilever model for piezoelectric energy harvesting from base excitations, Smart Mater. Struct. 18 (2) (2008) 18pp.

[14] R. Calio, U. Rongala, D. Camboni, M. Milazzo, C. Stefanini, G. de Petris, C. Oddo, Piezoelectric energy harvesting solutions, Biosensors 14 (3) (2014) 4755-4790.

[15] Y. Jia, A. A. Seshia, An auto-parametrically excited vibration energy harvester, Sens. Actuator A-Phys. 220 (2014) 69-75.

[16] Y. Jia, J. Yan, K. Soga, A. A. Seshia, Parametrically excited mems vibration energy harvesters with design approaches to overcome the initiation threshold amplitude, J. Micromech. Microeng 23 (11) (2013) 114007.

[17] Y. Jeon, R. Sood, J. h. Jeong, S.-G. Kim, Mems power generator with transverse mode thin film pzt, Sens. Actuator A-Phys. 122 (2005) 16-22.

[18] Y. Jia, A. Seshia, Power optimization by mass tuning for mems piezoelectric cantilever vibration energy harvesting, J Microelectromech. Syst. 25 (1) (2016) 108-117.

[19] Y. Jia, A. Seshia, Five topologies of cantilever-based mems piezoelectric vibration energy harvesters: a numerical and experimental comparison, Microsystems Technologies 22 (12) (2016) 2841-2852. 
[20] Y. Jia, A. Seshia, Directly and parametrically excited bi-stable vibration energy harvester for broadband operation, in: Transducers, Barcelona, Spain, 2013, pp. 454-457.

[21] Y. Jia, J. Yan, K. Soga, A. Seshia, Multi-frequency operation of a mems vibration energy harvester by accessing five orders of parametric resonance, in: J. Phys. Conf. Ser., Vol. 476 of 1, 2013, pp. 607-611.

[22] S. Li, A. Crovetto, Z. Peng, A. Zhang, O. Hansen, M. Wang, X. Li, F. Wang, Bi-resonant structure with piezoelectric pvdf films for energy harvesting from random vibration sources at low frequency, Sens. Actuator A-Phys. 247 (2016) $547-554$.

[23] E. Arroyo, Y. Jia, S. Du, S.-T. Chen, A. Seshia, Experimental and theoretical study of a piezoelectric vibration energy harvester under high temperature, $\mathrm{J}$ Microelectromech. Syst. 26 (6).

[24] S. Du, Y. Jia, A. Seshia, Maximizing output power in a cantilevered piezoelectric vibration energy harvester by electrode design, in: Journal of Physics: Conference Series, Vol. 660, IOP Publishing, 2015, p. 012114.

[25] S. Du, Y. Jia, S. Chen, C. Zhao, B. Sun, E. Arroyo, A. Seshia, A new electrode design method in piezoelectric vibration energy harvesters to maximize output power, Sens. Actuator A-Phys. 263 (2017) 693-701.

[26] Y. Jia, J. Yan, K. Soga, A. A. Seshia, Parametric resonance for vibration energy harvesting with design techniques to passively reduce the initiation threshold amplitude, Smart Mater. Struct. 23 (6) (2014) 13.

[27] A. G. A. Muthalif, N. H. D. Nordin, Optimal piezoelectric beam shape for single and broadband vibration energy harvesting: Modeling, simulation and experimental results, Mechanical Systems and Signal Processing 54-55 (2015) $417-426$.

[28] H. J. Song, Y.-T. Choi, N. M. Wereley, A. Purekar, Comparison of monolithic and composite piezoelectric material-based energy harvesting devices, Journal of Intelligent Material Systems and Structures 25 (14) (2014) 1825-1837.

[29] H. Sodano, Macro-fibre composites for sensing, actuation and power generation, Master's thesis, Virginia polytechnic institute and state university (2003). 
[30] Y. Shi, S. Hallett, M. Zhu, Energy harvesting behaviour for aircraft composites structures using macro-fibre composite: Part i-integration and experimental, Composite Structues 160 (2017) 1279-1286.

[31] A. Erturk, D. Inman, Issues in mathematical modeling of piezoelectric energy harvesters, Smart Mater. Struct. 17 (6) (2008) 14pp.

[32] A. Erturk, D. Inman, A distributed parameter electromechanical model for cantilevered piezoelectric energy harvesters, J. Vib. Acoust. 130 (4) (2008) $15 \mathrm{pp}$.

[33] A. Erturk, D. Inman, Piezoelectric energy harvesting, Wiley, New Delhi, India, 2011.

[34] S. Kundu, H. B. Nemade, Modeling and simulation of a piezoelectric vibration energy harvester, Procedia Engineering 144 (2016) 568-575.

[35] M. F. Daqaq, C. Stabler, Y. Qaroush, T. Seuaciuc-Osório, Investigation of power harvesting via parametric excitations, Journal of Intelligent Material Systems and Structures 20 (5) (2009) 545-557.

[36] Y. Shi, C. Piao, D. El-Fadlaoui, A. Al-Saadi, Y. Jia, Integration and characterisation of piezoelectric macro-fibre composite on carbon fibre composite for vibration energy harvesting, in: PowerMEMS, Daytona Beach, FL, USA, 2018.

[37] Smart Material, Mfc product properties [cited 23 November 2018]. URL www.smart-material.com/MFC-product-properties.html

[38] Do-160, environmental conditions and test procedures for airborne equipment, Tech. rep., Radio Technical Commission for Aeronautics (2010).

[39] J. R. Banerjee, Frequency equation and mode shape formulae for composite timoshenko beams, Composite Structures 51 (4) (2001) 381-388.

[40] A. G. Piersol, T. L. Paez, Shock and Vibrations Handbook, 6th Edition, McGraw Hill, 2009.

[41] Y. Jia, C. D. Do, X. Zou, A. A. Seshia, A hybrid vibration powered microelectromechanical strain gauge,, IEEE Sens. J. 16 (1) (2015) 235-241. 